\title{
Variation of Surface Topography in Laser Powder Bed Fusion Additive Manufacturing of Nickel Super Alloy 625
}

\author{
Jason C. Fox \\ National Institute of Standards and Technology, \\ Gaithersburg, MD 20899, USA \\ jason.fox@nist.gov \\ Data DOI: https://doi.org/10.18434/M32020 \\ Key words: additive manufacturing; focus variation; IN625; laser powder bed fusion; nickel super alloy 625; surface texture; surface \\ topography.
}

Accepted: September 5, 2019

Published: September 10, 2019

https://doi.org/10.6028/jres.124.023

\section{Summary}

This document provides details on the files available for download in the dataset "Variation of Surface Topography in Laser Powder Bed Fusion of Nickel Super Alloy 625." The following sections provide details on the experiments, methods, and data files. The experiment detailed in this document methodically varies part position and surface orientation relative to the build plate and relative to the recoater blade. This dataset provides surface height data for analysis and development of correlations by the greater research community.

\section{Data Specifications}

\begin{tabular}{ll}
\hline NIST Operating Unit(s) & Intelligent Systems Division, Engineering Laboratory \\
\hline Format & X3P \\
\hline \multirow{2}{*}{ Instrument } & An EOS M290 ${ }^{1}$ laser powder bed fusion system was used to \\
& fabricate the experiment samples. An Alicona InfiniteFocusXL200 \\
& G5 with Real3D Rotation Unit (focus variation microscope) was \\
\hline Spatial or Temporal Elements & used to measure surface heights on the experiment samples. \\
\hline
\end{tabular}

${ }^{1}$ Certain commercial equipment, instruments, or materials are identified in this paper in order to specify the experimental procedure adequately. Such identification is not intended to imply recommendation or endorsement by the National Institute of Standards and Technology, nor is it intended to imply that the materials or equipment identified are necessarily the best available for the purpose. 


\begin{tabular}{ll}
\hline Data Dictionary & https://github.com/OpenFMC/x3p \\
\hline Accessibility & $\begin{array}{l}\text { All datasets }{ }^{2} \text { submitted to Journal of Research of NIST are publicly } \\
\text { available. }\end{array}$ \\
\hline License & https://www.nist.gov/director/licensing \\
\hline
\end{tabular}

\section{Experiment Method}

For this work, a single additive manufacturing build with nine artifacts was carried out using an EOS M290 laser powder bed fusion system. The artifacts were built in a nickel super alloy 625 using EOS NickelAlloy IN625 powder [1]. All artifacts were built using the process parameters listed in Table 1 through Table 3, and vendor specified procedures were followed.

Table 1. Process parameters used for the build.

\begin{tabular}{|c|c|}
\hline Parameter Settings & Value \\
\hline Pre-exposure type & No Exposure \\
\hline Skin exposure type & See Table 2 \\
\hline Core exposure type & No Exposure \\
\hline Post exposure type & See Table 3 \\
\hline Skin thickness (x/y): & $200.00 \mathrm{~mm}$ \\
\hline Skin thickness (z): & $100.00 \mathrm{~mm}$ \\
\hline Beam radius: & $0.00 \mathrm{~mm}$ \\
\hline Core open to platform & Unchecked \\
\hline Skin/core & Checked \\
\hline
\end{tabular}

\footnotetext{
${ }^{2}$ The National Institute of Standards and Technology (NIST) uses its best efforts to deliver a high-quality copy of the Database and to verify that the data contained therein have been selected on the basis of sound scientific judgment. However, NIST makes no warranties to that effect, and NIST shall not be liable for any damage that may result from errors or omissions in the Database.
} 


\section{Journal of Research of the National Institute of Standards and Technology}

Table 2. Skin exposure type settings.

\begin{tabular}{|c|c|c|}
\hline Skin Exposure Type Tab & Setting & Value \\
\hline \multirow{12}{*}{ Stripes } & Distance & $0.11 \mathrm{~mm}$ \\
\hline & Speed & $960.0 \mathrm{~mm} / \mathrm{s}$ \\
\hline & Power & $285.0 \mathrm{~W}$ \\
\hline & Beam offset & $0.015 \mathrm{~mm}$ \\
\hline & Stripe width & $10.00 \mathrm{~mm}$ \\
\hline & Stripes overlap & $0.08 \mathrm{~mm}$ \\
\hline & Skywriting & Checked \\
\hline & Offset & Checked \\
\hline & Hatching: X & Checked \\
\hline & Hatching: Y & Checked \\
\hline & Hatching: Alternating & Checked \\
\hline & Hatching: Rotated & Checked \\
\hline \multirow{17}{*}{ UpDown } & Upskin: Distance & $0.09 \mathrm{~mm}$ \\
\hline & Upskin: Speed & $600.0 \mathrm{~mm} / \mathrm{s}$ \\
\hline & Upskin: Power & $153.0 \mathrm{~W}$ \\
\hline & Upskin: Thickness & $0.12 \mathrm{~mm}$ \\
\hline & Upskin: X & Checked \\
\hline & Upskin: Y & Checked \\
\hline & Upskin: Alternating & Unchecked \\
\hline & Downskin: Distance & $0.05 \mathrm{~mm}$ \\
\hline & Downskin: Speed & $2400.0 \mathrm{~mm} / \mathrm{s}$ \\
\hline & Downskin: Power & $145.0 \mathrm{~W}$ \\
\hline & Downskin: Thickness & $0.16 \mathrm{~mm}$ \\
\hline & Downskin: $\mathrm{X}$ & Checked \\
\hline & Downskin: Y & Checked \\
\hline & Downskin: Alternating & Checked \\
\hline & Overlap with inskin & $0.10 \mathrm{~mm}$ \\
\hline & Min. length & $0.10 \mathrm{~mm}$ \\
\hline & Skywriting & Checked \\
\hline \multirow{3}{*}{ Skip layer } & Skipped layers & 0 \\
\hline & Offset layers & 0 \\
\hline & Expose first layer & Checked \\
\hline
\end{tabular}


Table 3. Postcontour exposure type settings.

\begin{tabular}{|c|c|c|}
\hline Postcontour Exposure Type Tab & Setting & Value \\
\hline \multirow{11}{*}{ Contour (first tab) } & Standard: Speed & $300.0 \mathrm{~mm} / \mathrm{s}$ \\
\hline & Standard: Power & $138.0 \mathrm{~W}$ \\
\hline & On Part: Speed & $300.0 \mathrm{~mm} / \mathrm{s}$ \\
\hline & On Part: Power & $138.0 \mathrm{~W}$ \\
\hline & Downskin: Speed & $1400.0 \mathrm{~mm} / \mathrm{s}$ \\
\hline & Downskin: Power & $140.0 \mathrm{~W}$ \\
\hline & Contour & Checked \\
\hline & Post contour & Checked \\
\hline & Beam offset & $0.012 \mathrm{~mm}$ \\
\hline & Thickness & $0.040 \mathrm{~mm}$ \\
\hline & Corridor & $0.040 \mathrm{~mm}$ \\
\hline \multirow{11}{*}{ Contour (second tab) } & Standard: Speed & $800.0 \mathrm{~mm} / \mathrm{s}$ \\
\hline & Standard: Power & $80.0 \mathrm{~W}$ \\
\hline & On Part: Speed & $800.0 \mathrm{~mm} / \mathrm{s}$ \\
\hline & On Part: Power & $80.0 \mathrm{~W}$ \\
\hline & Downskin: Speed & $1600.0 \mathrm{~mm} / \mathrm{s}$ \\
\hline & Downskin: Power & $80.0 \mathrm{~W}$ \\
\hline & Contour & Checked \\
\hline & Post contour & Checked \\
\hline & Beam offset & $0.000 \mathrm{~mm}$ \\
\hline & Thickness & $0.040 \mathrm{~mm}$ \\
\hline & Corridor & $0.040 \mathrm{~mm}$ \\
\hline \multirow{8}{*}{ Edges } & Edge factor & 2.00 \\
\hline & Threshold & 3.0 \\
\hline & Min radius factor & 0.00 \\
\hline & Beam offset & $0.000 \mathrm{~mm}$ \\
\hline & Speed & $900.0 \mathrm{~mm} / \mathrm{s}$ \\
\hline & Power & $100.0 \mathrm{~W}$ \\
\hline & Edges & Checked \\
\hline & Post edge & Checked \\
\hline
\end{tabular}

An example of the artifact can be seen in Fig. 1. The artifact was designed to be $38.18 \mathrm{~mm}$ tall and $45.18 \mathrm{~mm}$ wide. The part has a $7 \mathrm{~mm}$ diameter hole, vertically through the center of the part, that was used for mounting the sample within the measurement equipment.

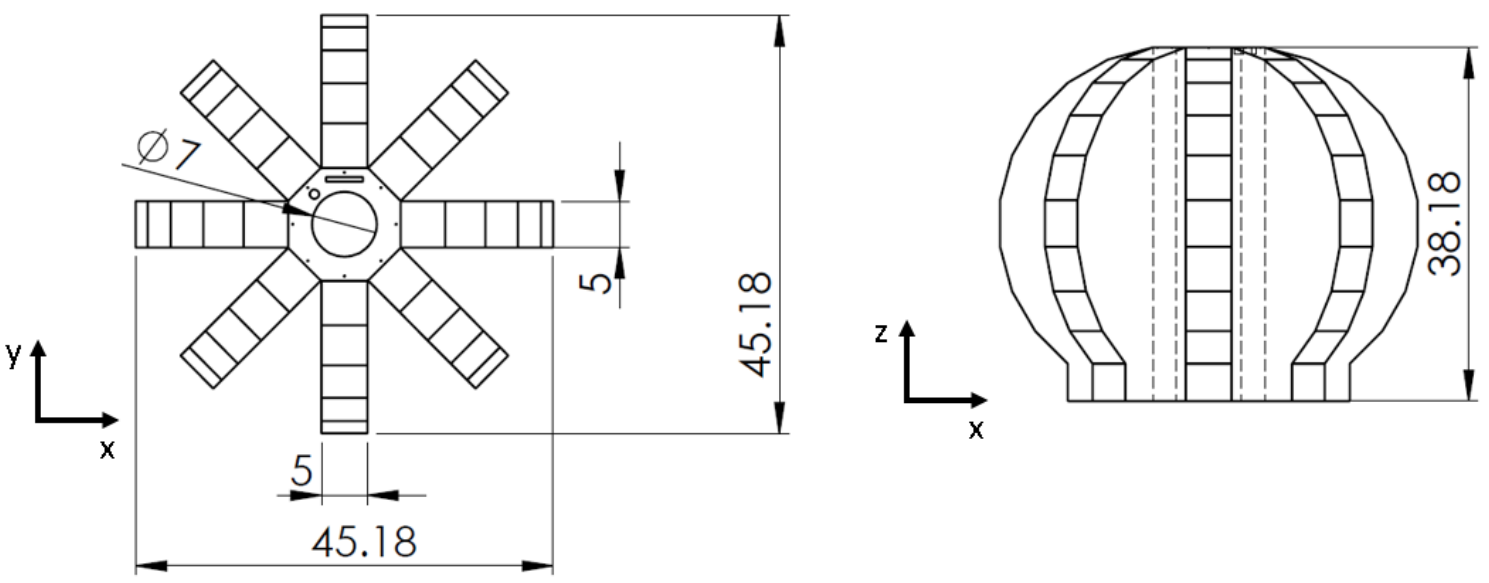

Fig. 1. Schematic of the test artifact. Dimensions are in millimeters. 
Each artifact has eight ribs and nine surfaces per rib, as seen in Fig. 2. Ribs are evenly spaced at $45^{\circ}$ intervals. The artifact was designed such that each surface is a planar $5 \mathrm{~mm}$ x $5 \mathrm{~mm}$ area. Surface angles relative to the build plate are listed in Table 4 . As an example, downward facing surfaces will have an angle less than $90^{\circ}$, upward facing surfaces will have an angle greater than $90^{\circ}$, and a vertical surface will have an angle equal to $90^{\circ}$.
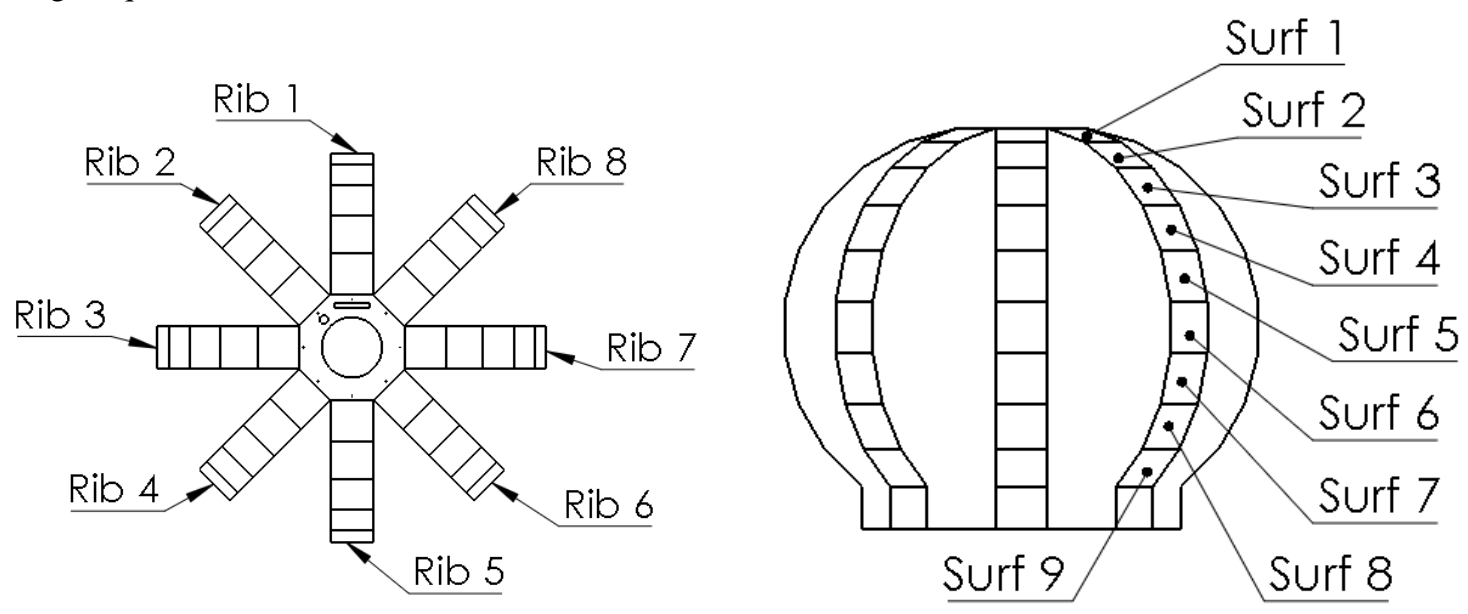

Fig. 2. Identification of ribs and surfaces of the artifact.

Table 4. Angle of each surface as measured from the build plate.

\begin{tabular}{|c|c|}
\hline Surface & Angle $\left.\mathbf{(}^{\circ}\right)$ \\
\hline Surf 1 & 165 \\
\hline Surf 2 & 150 \\
\hline Surf 3 & 135 \\
\hline Surf 4 & 120 \\
\hline Surf 5 & 105 \\
\hline Surf 6 & 90 \\
\hline Surf 7 & 75 \\
\hline Surf 8 & 60 \\
\hline Surf 9 & 45 \\
\hline
\end{tabular}

For the build, all parts are oriented such that Rib 1 faces the back of the machine and Rib 5 faces the front of the machine. Coordinate positions of parts within the build volume is based on ISO/ASTM 52921:2013(E) [2]. Positions of parts are listed in Table 5. The sample names in Table 5 are abbreviated STV for surface texture variability. Renderings of the layout are shown in Fig. 3 and Fig. 4. The resultant build and one of the artifacts can be seen in Fig. 5. Note that four $10 \mathrm{~mm}$ x $10 \mathrm{~mm}$ x $40 \mathrm{~mm}$ cuboids were built during this experiment and can be seen in Fig. 3 through Fig. 5. These samples, however, were not measured as part of this research and are not included in the dataset. 
Table 5. Coordinate positions of parts within the build volume.

\begin{tabular}{|l|c|}
\hline Sample Name & Location $(\mathbf{X}, \mathbf{Y}, \mathbf{Z}) \mathbf{~ m m}$ \\
\hline STV1 & $-75,75,0$ \\
\hline STV2 & $0,75,0$ \\
\hline STV3 & $75,75,0$ \\
\hline STV4 & $-75,0,0$ \\
\hline STV5 & $0,0,0$ \\
\hline STV6 & $75,0,0$ \\
\hline STV7 & $-75,-75,0$ \\
\hline STV8 & $0,-75,0$ \\
\hline STV9 & $75,-75,0$ \\
\hline
\end{tabular}

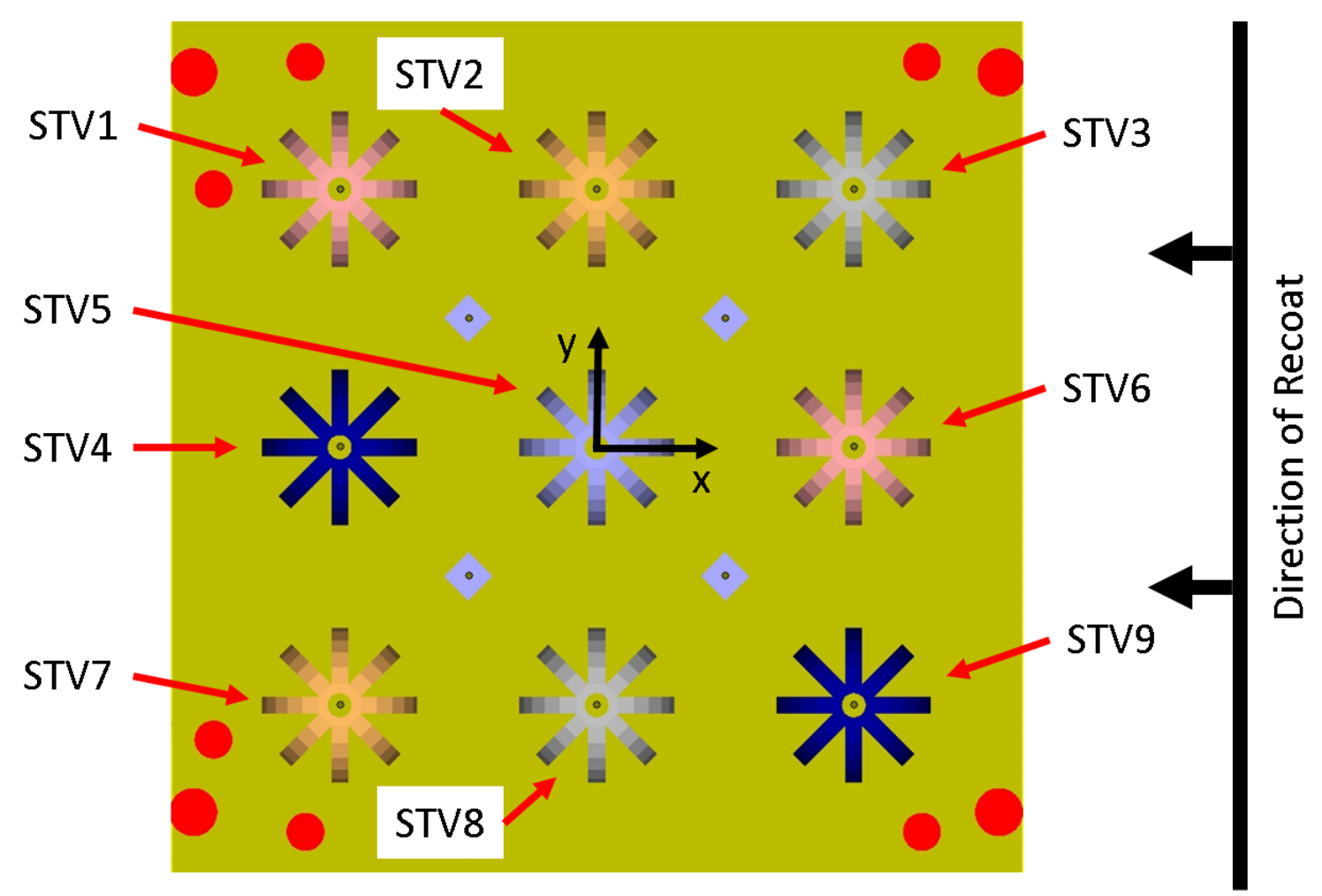

\section{Front of Machine}

Fig. 3. Top view rendering of the part positioning. Note the origin $(0,0,0)$ is on the build plate in the center of the STV5. Direction of $\mathrm{z}$-axis in the above figure is based on the right-hand rule. Red circles indicate mounting locations for the build plate. Blue rectangles indicate test parts not used in this study. 


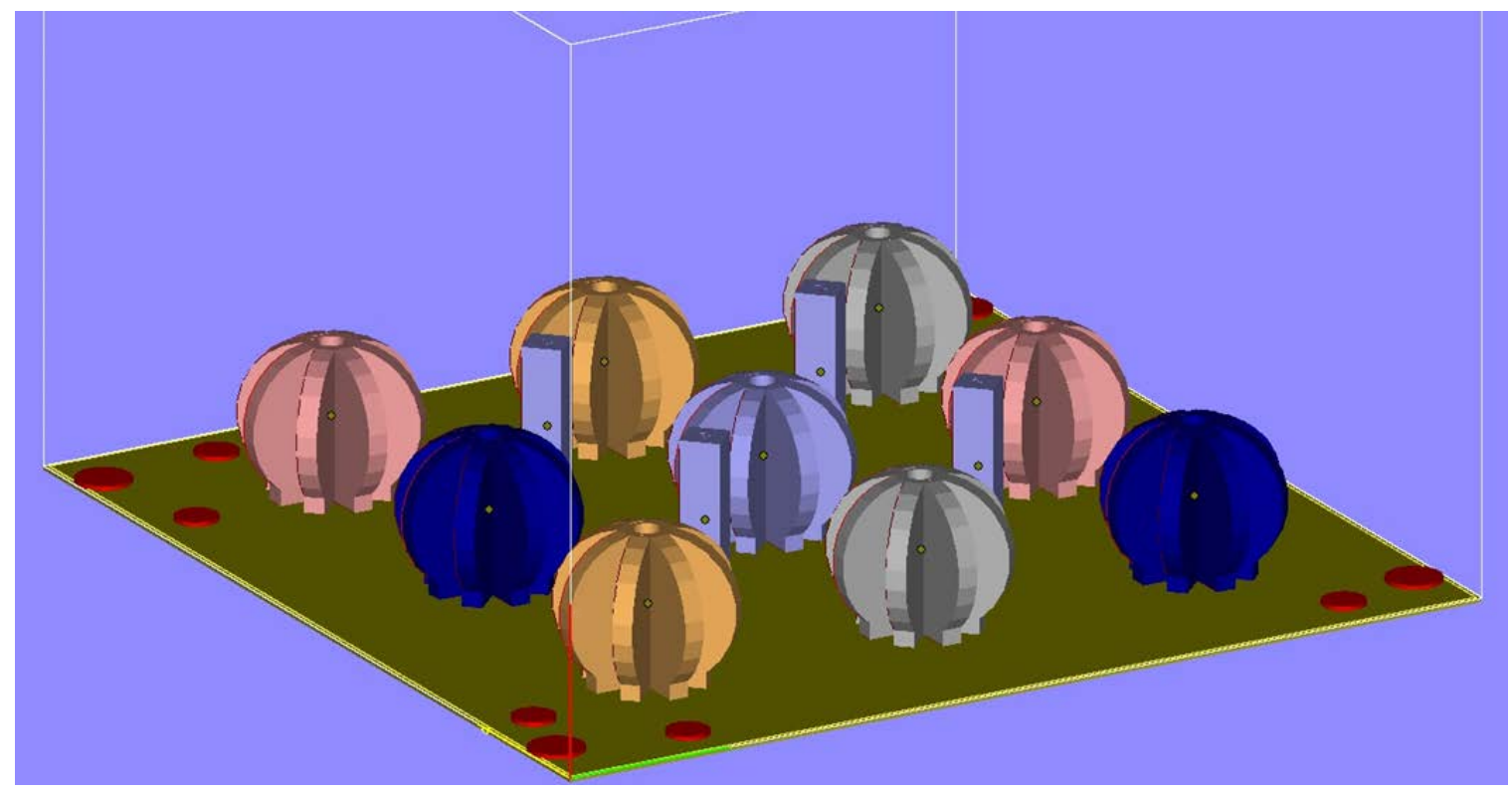

Fig. 4. Perspective view rendering of the build layout.
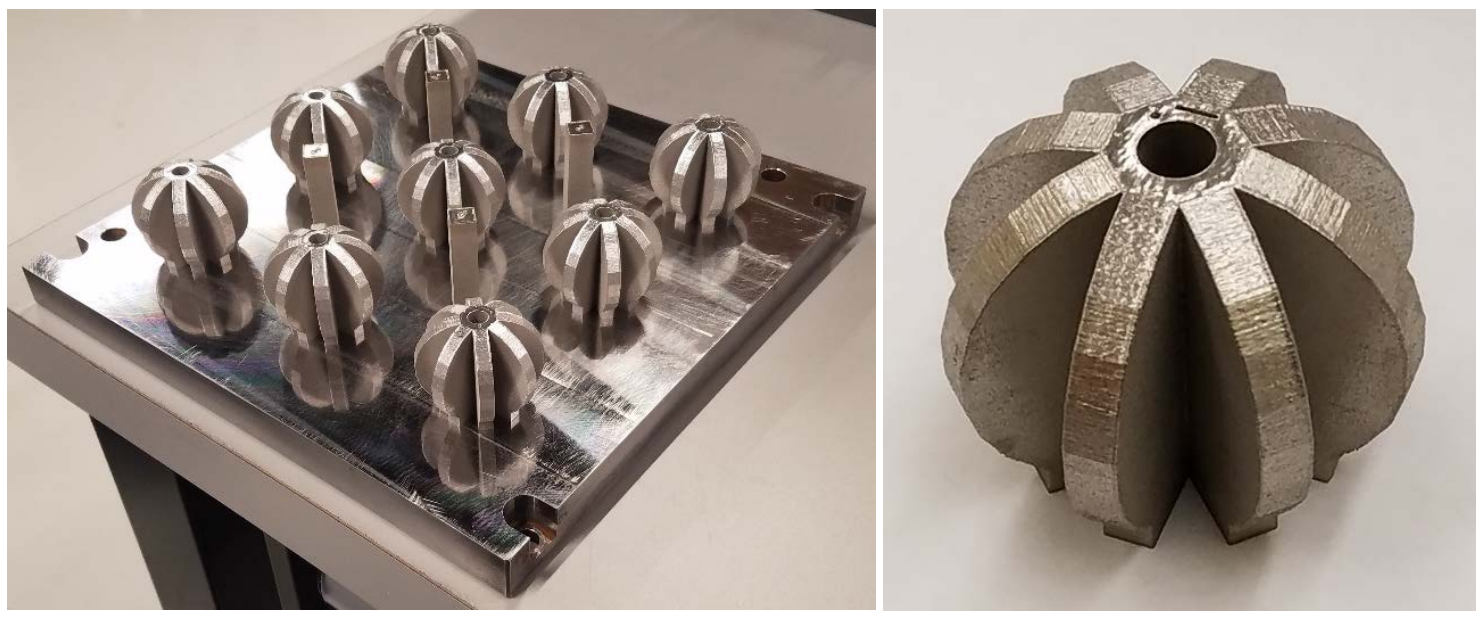

Fig. 5. Image of the build (left) and one of the artifacts after it was removed from the build plate (right).

Parts were removed from the build plate using wire electrical discharge machining (EDM). To remove contamination from the wire EDM process and loosely attached powder particles, all parts were cleaned in an ultrasonic bath of acetone for 5 minutes, rinsed with ethanol, and allowed to air dry. Following the cleaning procedure, powder-free gloves were used to handle the parts to prevent residue or oils from skin depositing on the surfaces to be measured. At no point were towels, cloths, wipes, etc. used to dry, clean, or handle the parts to prevent fibers from attaching to the surfaces to be measured. 


\section{Measurement Method and Data Processing}

Measurements were performed using an Alicona InfiniteFocus G5 focus variation microscope [3] with Real3DRotation unit, seen in Fig. 6. For ease of measurement, the artifact was attached to a bolt that fed through the center hole of the artifact and then held in the microscope's rotation unit. An example of the mounted sample can also be seen in Fig. 6. This allowed for tilt and rotation of the part so that measurements of each surface were made with the objective axis of the microscope normal to the surface. Measurements were performed with the 20x objective with both coaxial and ring (off-axis) lighting. Light settings were adjusted for each surface to minimize data dropout in the measurement. Lateral resolution setting of the microscope was set to $1.5 \mu \mathrm{m}$ and point spacing was adjusted to $0.5 \mu \mathrm{m}$. Vertical resolution was set to $0.1 \mu \mathrm{m}$. Surface height maps were created by the microscope software by stitching an $8 \mathrm{x} 8$ fieldof-view (FoV) set of measurements together.

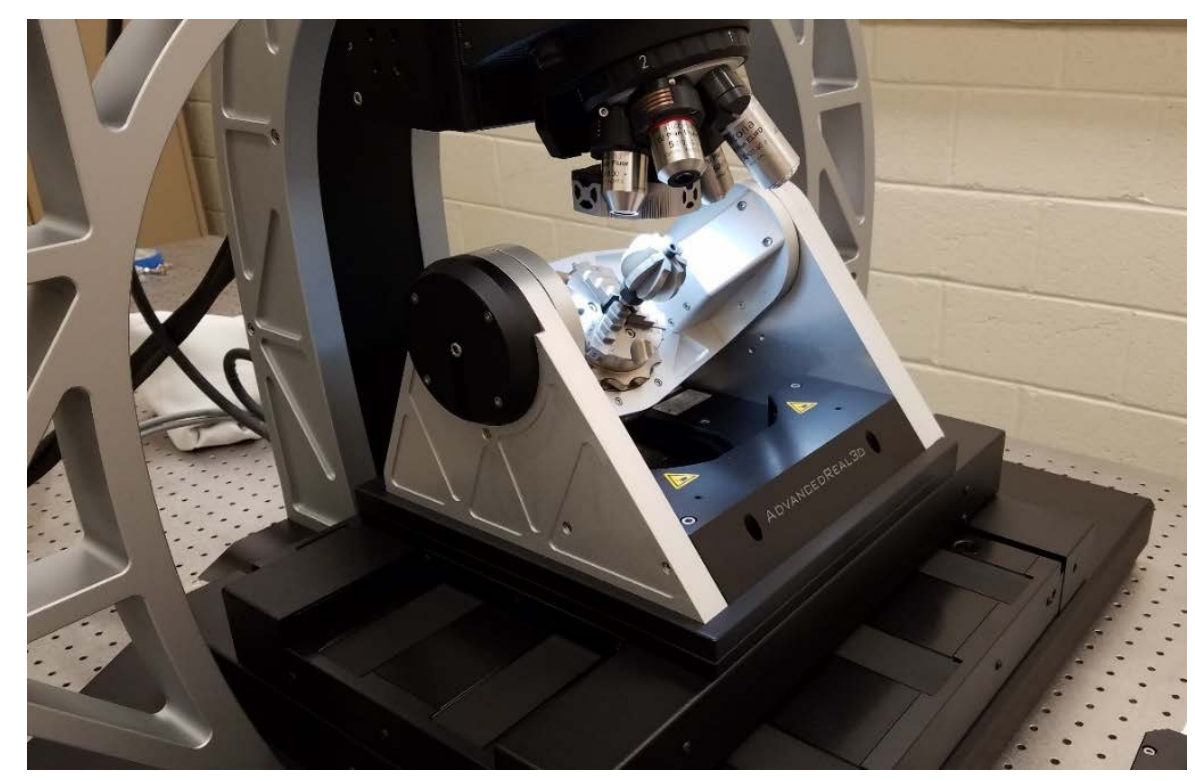

Fig. 6. Example part mounted in the microscope for measurement.

All data has been cropped to remove edges of the parts from the uploaded dataset. To perform this operation, data was exported from the microscope's software in an X3P data format and loaded into MATLAB for processing. Information on the X3P data format and MATLAB tools for importing/exporting X3P data are publicly available via the OpenFMC repository [4]. In MATLAB, the center position of the dataset is determined in two steps. First, the center position along the $\mathrm{x}$ axis is determined using the halfway point between the data dropout on the left and right side of the dataset. This is done automatically by averaging the z-values in the dataset for the middle $1000 \mathrm{y}$-locations and determining the x-locations where the data returns a null value, as shown in Fig. 7. 

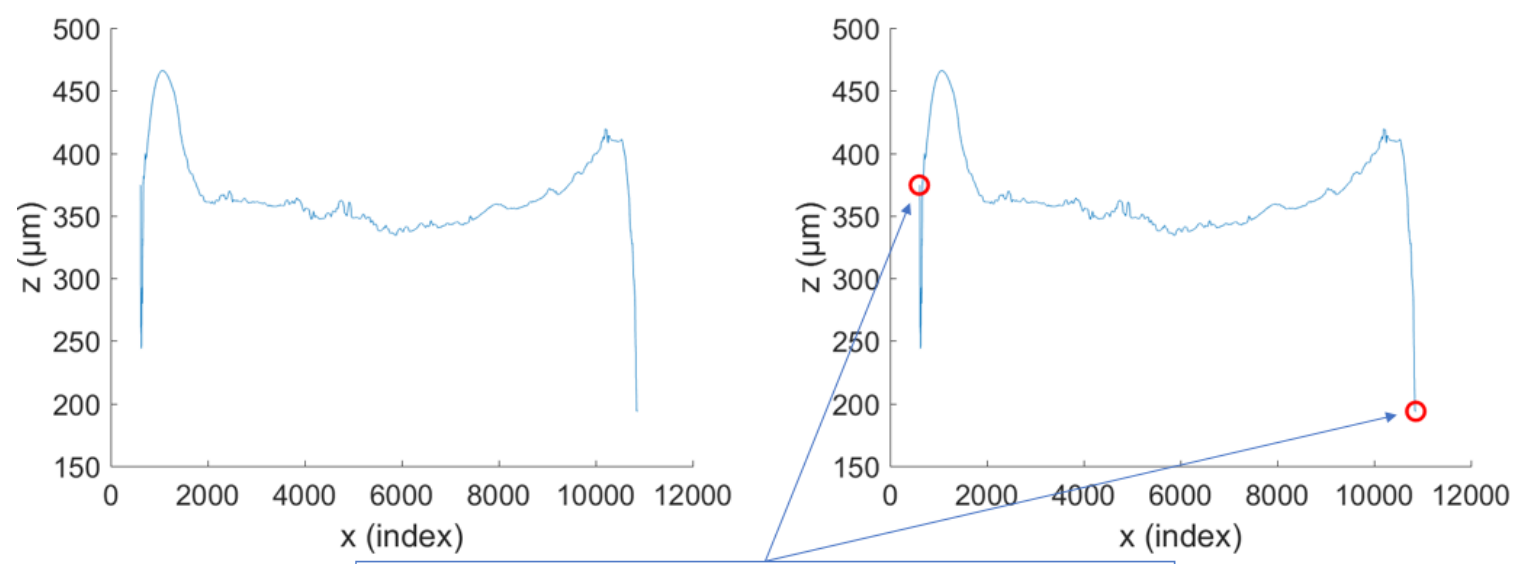

These signify the edges of the part and the center will be the average of these two $x$ positions

Fig. 7. Depiction of how the center position of the surface along $x$ is determined via automatic selection of data dropout.

Second, the center position along the y-axis must be determined manually. This is because the surface being measured connects to the other surfaces on the rib in the y direction. Thus, the average 1000 points along the $\mathrm{x}$-axis are presented for manual interpretation and the edges of the surface are determined by the last peak available before the data drops off significantly, as shown in Fig. 8.

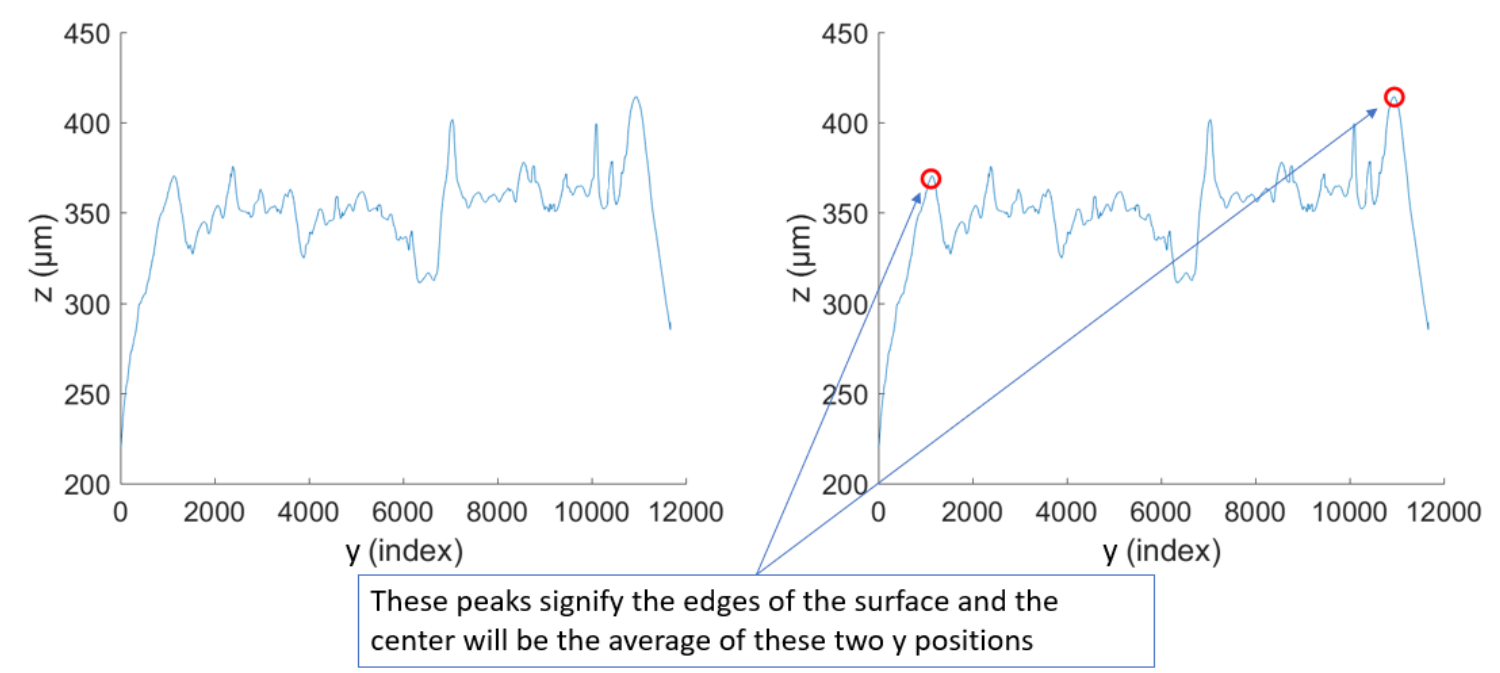

Fig. 8. Depiction of how the center position along y is determined via manual selection of peaks.

This method results in a center position and the resultant cropped surface is a $4 \mathrm{~mm}$ by $4 \mathrm{~mm}$ area about the center position, shown in Fig. 9. 


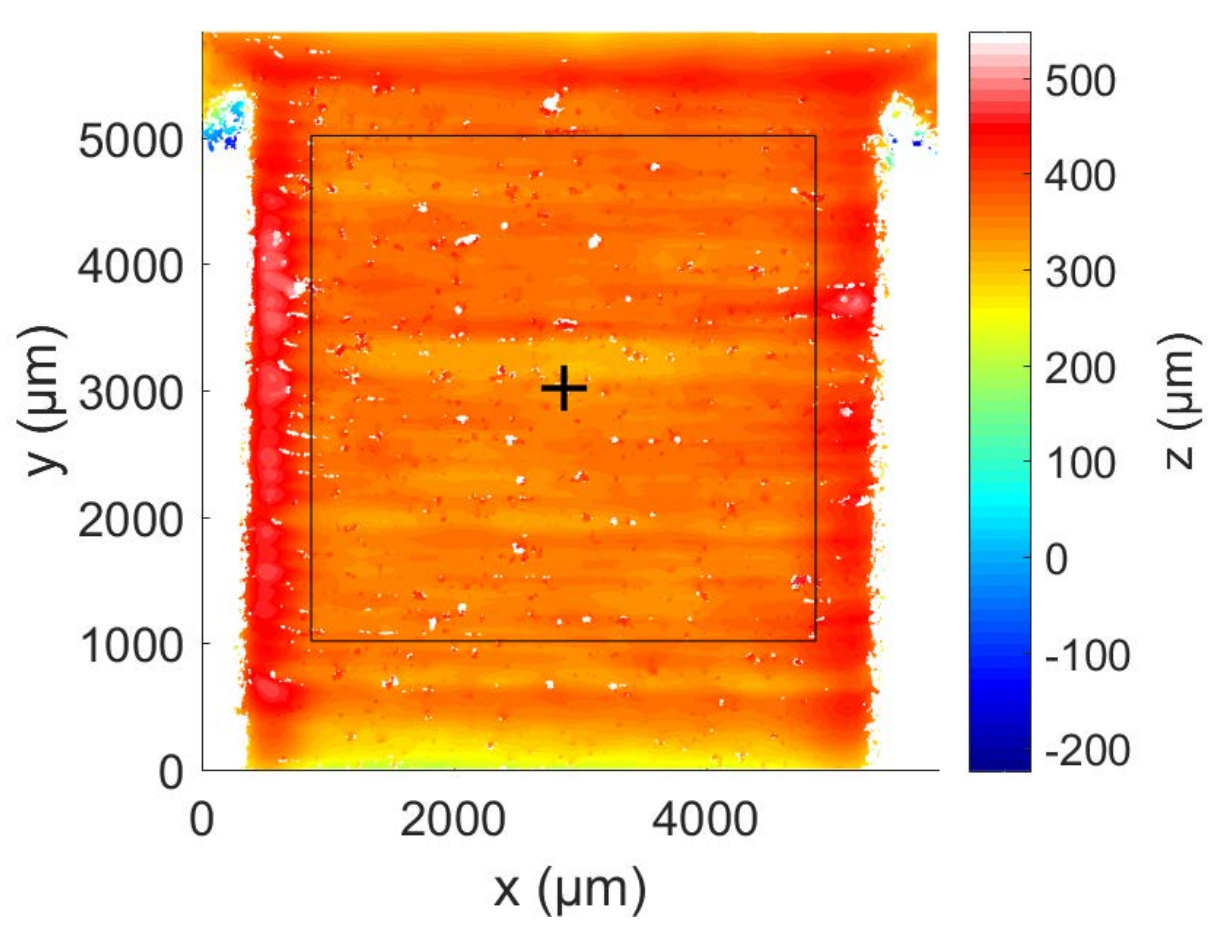

Fig. 9. Example of surface with center position and cropped region highlighted.

Surface data is stored using the X3P data format [4]. No adjustments to the data aside from the cropping described previously were performed (i.e., no filtering, no adjustments to tilt, no outlier removals). All surface data files are set such that the build direction of the part is aligned with the positive $y$ direction of the measurement data and the bottom left corner of the surface is at $(x, y)=(0,0)$. Surface data was transposed to ensure the build direction matches this description. No interpolation was performed to achieve this orientation, only transposing/flipping of the arrays when necessary as the build direction was already aligned with the $y$-axis of the microscope. Note that the ISO standard defining the X3P data format has been revised in the past and is currently under review for additional revisions. To avoid ambiguity in the orientation of the surface files, an orientation data set has been included. Data from the 'orientation data.x3p' file should load such that the number "75" is legible in the data set when plotted with the bottom left corner being $(\mathrm{x}, \mathrm{y})=(0,0)$, as seen in Fig. 10 . 


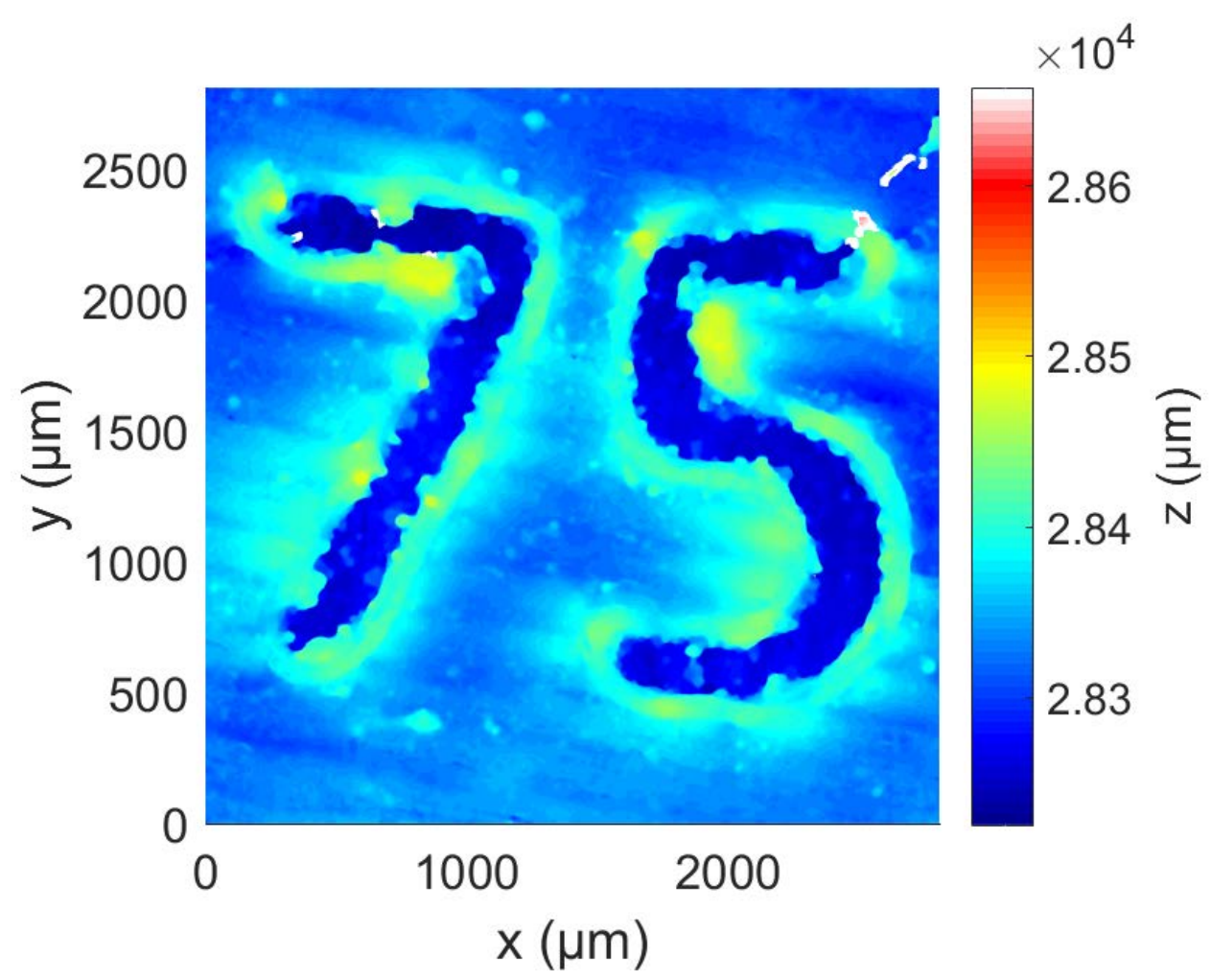

Fig. 10. View of the orientation data set 'orientation data.x3p'.

\section{Data Files}

The dataset contains the following files:

- 20180901_STV_LPBF_IN625.pdf

o This dataset overview document

- 'Orientation and Sample Data.zip'

0 'orientation data.x3p'

- An orientation dataset described in Section 4.

o 'STV5 Rib 1 Surf 1 subset-t.x3p'

- Surface data from STV5 Rib 1 Surface 1. This data is also included in the 'STV5.zip' file but was included here for users to quickly access data and better understand the dataset.

- $\quad$ STV1.zip

0 A collection of X3P data files from the part 'STV1'

- $\quad$ STV2.zip

0 A collection of X3P data files from the part 'STV2'

- $\quad$ STV3.zip

o A collection of X3P data files from the part 'STV3'

- $\quad$ STV4.zip

o A collection of X3P data files from the part 'STV4'

- $\quad$ STV5.zip 


\author{
o A collection of X3P data files from the part 'STV5' \\ - $\quad$ STV6.zip \\ o A collection of X3P data files from the part 'STV6' \\ - $\quad$ STV7.zip \\ o A collection of X3P data files from the part 'STV7' \\ - $\quad$ STV8.zip \\ o A collection of X3P data files from the part 'STV8' \\ - $\quad$ STV9.zip \\ o A collection of X3P data files from the part 'STV9'
}

Note that all surface X3P files are denoted by part name (e.g., STV1, STV2, etc.), rib number (e.g., Rib 1, Rib 2, etc.), and surface number (e.g., Surf 1, Surf 2, etc.). Each file also has a 'subset-t' identifier to indicate that it is a subset of the full measurement and transformed to align the build direction with the positive y-direction, as described in Section 4. Thus, surface 1 on rib 2 of part STV3 will have the file name 'STV3 Rib 2 Surf 1 subset-t.x3p'.

All surface files (i.e., .X3P files), aside from the orientation file, contain a $4 \mathrm{~mm}$ by $4 \mathrm{~mm}$ area of $\mathrm{z}$ heights at a point spacing of $0.5 \mu \mathrm{m}$ in both $\mathrm{x}$ and $\mathrm{y}$. All surface X3P files are in units of $\mu \mathrm{m}$.

\title{
6. Impact
}

This dataset is for the exploration and development of correlations between build position and orientation, and surface topography. All too often, data provided in research for the analysis of additive manufacturing (AM) surface topography lacks adequate description of process parameters used to build and measure experiment samples. Additionally, the development of correlations requires large quantities of data due to the numerous variables that may affect surface topography, which can be time consuming and/or cost prohibitive. The dataset provided attempts to address these issues and users are encouraged to explore advanced filtering, segmentation, and other techniques to help identify correlations.

\section{Acknowledgments}

The author would like to acknowledge Dr. Alkan Donmez, Dr. Jarred Heigel, Dr. Felix Kim, and Dr. Anne-Francois Obaton for their input on the design of the artifact and design of the experiment; Jared Tarr for his assistance with the build and measurements; and internship students Mathieu Lacourciere and Ethan Martinez for their assistance preparing data files.

\section{References}

[1] Material Data Sheet - EOS NickelAlloy IN625 2011. http://ip-saas-eoscms.s3.amazonaws.com/public/d1327facdca0e32a/373a60ec4f5c891b7dbcdf572e37d3b0/EOS_NickelAlloy_IN625_en.pdf (accessed June 13, 2017).

[2] ASTM ISO/ASTM52921-13 Standard Terminology for Additive Manufacturing-Coordinate Systems and Test Methodologies, ASTM International, West Conshohocken, PA, 2013, https://doi.org/10.1520/ISOASTM52921-13

[3] ISO 25178-606:2015. Geometrical product specification (GPS) -- Surface texture: Areal -- Part 606: Nominal characteristics of non-contact (focus variation) instruments 2015.

[4] The OpenFMC repository for C/C++ and other code for reading and writing X3P files.: OpenFMC/x3p 2018. https://github.com/OpenFMC/x3p (accessed September 26, 2018). 
About the author: Jason C. Fox, Ph.D. is a mechanical engineer in the Intelligent Systems Division at NIST. Jason has been studying additive manufacturing processes since 2010. He has primarily focused on measuring and understanding the surface topography of AM parts to better aid inspection and qualification. As part complexity increases, the ability to perform post build treatments to improve the surface finish of AM parts decreases. It is therefore imperative that we understand the range of surface topographies present in AM and how it effects part quality. In addition to investigations of surface topography, Jason has developed thermal finite element models of the additive process to understand melt pool geometry and the transient response of the melt pool to changes in process parameters. Jason earned a Ph.D. in mechanical engineering from Carnegie Mellon University in 2015.

The National Institute of Standards and Technology is an agency of the U.S. Department of Commerce. 